\title{
COMPUTER SIMULATION OF SPATIAL ARRANGEMENT AND CONNECTIVITY OF PARTICLES IN THREE-DIMENSIONAL MICROSTRUCTURE: APPLICATION TO MODEL ELECTRICAL CONDUCTIVITY OF POLYMER MATRIX COMPOSITE
}

\author{
P. LOUIS' and A. M. GOKHALE \\ 'Aerospatiale, Centre Commun De Recherche, 12 Rue Pasteur, BP 76, 92152 Suresnes Cedex, France and \\ ${ }^{2}$ School of Materials Science and Engineering, Georgia Institute of Technology, Atlanta, \\ GA 30332-0245, U.S.A.
}

(Received 27 February 1995; in revised form 21 June 1995)

\begin{abstract}
Computer simulation is a powerful tool for analyzing the geometry of three-dimensional microstructure. A computer simulation model is developed to represent the three-dimensional microstructure of a two-phase particulate composite where particles may be in contact with one another but do not overlap significantly. The model is used to quantify the "connectedness" of the particulate phase of a polymer matrix composite containing hollow carbon particles in a dielectric polymer resin matrix. The simulations are utilized to estimate the morphological percolation volume fraction for electrical conduction, and the effective volume fraction of the particles that actually take part in the electrical conduction. The calculated values of the effective volume fraction are used as an input for a self-consistent physical model for electrical conductivity. The predicted values of electrical conductivity are in very good agreement with the corresponding experimental data on a series of specimens having different particulate volume fraction.
\end{abstract}

\section{INTRODUCTION}

Physical properties of multi-phase solids often depend on the arrangement of features in threedimensional microstructural space. For example, fluid flow through a porous solid depends on the connectivity of the pores [1]. Similarly, electrical conductivity of a two-phase composite containing particles of a conductive material in a dielectric matrix critically depends on "connectedness" of the conducting particulate phase.

Two important aspects of the spatial arrangement of the microstructural features are: (j) distribution of distances between particles (or features); and (ii) "connectedness" of the microstructural features in three-dimensional microstructural space. Several rigorously defined and physically meaningful descriptors of inter-particle distance distributions are available (for example, nearest neighbor distribution function, radial distribution function, etc. $(2-4])$. The choice of a specific descriptor obviously depends on the physical phenomena or property under investigation. Recently, an efficient and practical digital image analysis technique has been developed for statistically reliable estimation of inter-particle distance distributions observed in a metallographic plane [5]. The three-dimensional inter-particle distance distributions can be estimated from the corresponding planar data under certain conditions $[6,7]$. On the other hand, practical experimental techniques are not available for estimation of the attributes related to the "connectedness" of microstructural features in 3D space, from the observations on the metallographic sections. Further. the attributes of the "connectedness" that are of relevance to the phenomena, such as electrical conduction, are not well understood. In this context. the topological connectivity of the particle surfaces (i.e. genus or first Betti number) may not represent the relevant aspect of the particle connectivity. This leads to difficulties in establishing meaningful quantitative correlations between the microstructure and electrical conductivity of two-phase (or multi-phase) solids containing particulate of a conducting phase in a dielectric matrix of anothy phase.

Computer simulation of three-dimensional microstructures is an attractive alternative for studying the spatial arrangement of particles (features). and for developing the microstructural parameter(s) that represents the relevant aspects of the "connectedness". A microstructure based physical model for electrical conductivity of two-phase solids can be developed from the resulting information. This paper reports a new computer simulated model of three-dimensional microstructure containing poly. dispersed spherical particles that may be in mutual

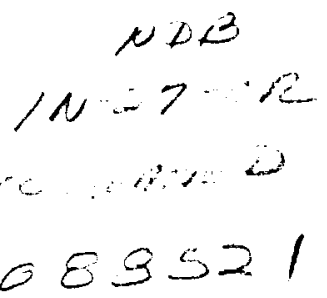


contact, but do not overlap significantly. The simulation is used to predict the percolation threshold for the formation of continuous particle chains, to compute the volume fraction of the particles that participate in the electrical conduction (called effective volume fraction), and to develop a simple selfconsistent model for the electrical conductivity of the polymer matrix composite containing a population of hollow spherical carbon particles in the dielectric polymeric matrix of Araldite. The predictions of the model are in good agreement with the experimental data on electrical conductivity of a series of specimens that have different volume fraction of the particulate phase, but the same particle size frequency function. The aspects of the "connectedness" of the carbon particles that are of relevance to the electrical conduction are represented by effective particulate volume fraction, which is simply the volume fraction of the particles that contribute to the electrical conduction. The global particulate volume fraction and the particle size distribution function are the input for the computer simulation of the three-dimensional microstructure. Experimental measurements of the nearest neighbor distribution and radial distribution function of particles present in a random metallographic plane, obtained from digital image analysis, are in good agreement with the corresponding attributes of a random two-dimensional plane through simulated three-dimensional microstructure, demonstrating that the simulated microstructures are a reasonable representation of actual microstructures.

A brief background on the characterization of spatial arrangements of particles and computer simulation of microstructures is given in the next section. The new computer simulated microstructure model and experimental work are presented in the subsequent sections. Finally, the self-consistent microstructure based physical model for electrical conductivity of a two-phase solid is presented, and the predictions of this model are compared with the experimental data on electrical conductivity.

\section{BACKGROUND}

\section{Computer simulation of microstructures}

For computer simulation of any modeled microstructure, the first step is the representation of the microstructural space by a set of regularly and closely spaced discrete coordinate points $\left(X_{i}, Y_{i}, Z_{i}\right)$. This is equivalent to the generation of a three-dimensional "graph paper". The total number of coordinate points depends on the volume of the space to be simulated, and the distance between the consecutive points. Once the microstructural space is simulated, the particles (or features) are placed in this "box" one by one: the size, shape and location of the particles are chosen according to a set of rules that make up numbers (say, $1-n, 1-m$ and $1-p$ ), three numbers (one from each set) are chosen at random. These three numbers (say, $X_{0}, Y_{0}, Z_{0}$ ) constitute the coordinates of the center of the first sphere. Once the radius of this sphere (say, $R_{0}$ ) is specified (according to some fixed scheme), the equation of this sphere is known, and the first particle is essentially placed in the box. One now chooses the center and the radius of the second "probable" particle by using the same scheme. From the known equations of the surfaces of the first and the second sphere, it can be detected if these two spheres intersect. If they intersect, then the second particle is rejected, new center coordinates and radius are chosen using the same algorithm, and the process is repeated till the second particle is located such that it does not intersect the first one. Using the same logic, the third particle is chosen so that it does not intersect the first two particles. The process is repeated till the space is filled with the required volume fraction of the spheres having preset size distribution. The microstructure is thus represented by a set of equations of the surfaces of non-overlapping spheres. Figure 1 shows a schematic two-dimensional microstructure generated by the "hard sphere" (i.e. hard "circles" in 2D) model. The "hard sphere" model is useful for simulating a microstructure consisting of uniformly distributed spherical particles that do not touch one another. Another popular microstructure model is the Boolean model, where randomly distributed convex particles (for example, spheres) are allowed to freely overlap to any extent [9]. Figure 2 shows a schematic representation of a two-dimensional microstructure generated by the Boolean model. Several more sophisticated models are available in the literature $[10,11]$. However, such models are designed for specific applications, and they require powerful mainframe computers.

The particulate volume fraction and size distribution are input parameters for all the simulated microstructures. It follows that the microstructures simulated from different models differ mainly in the

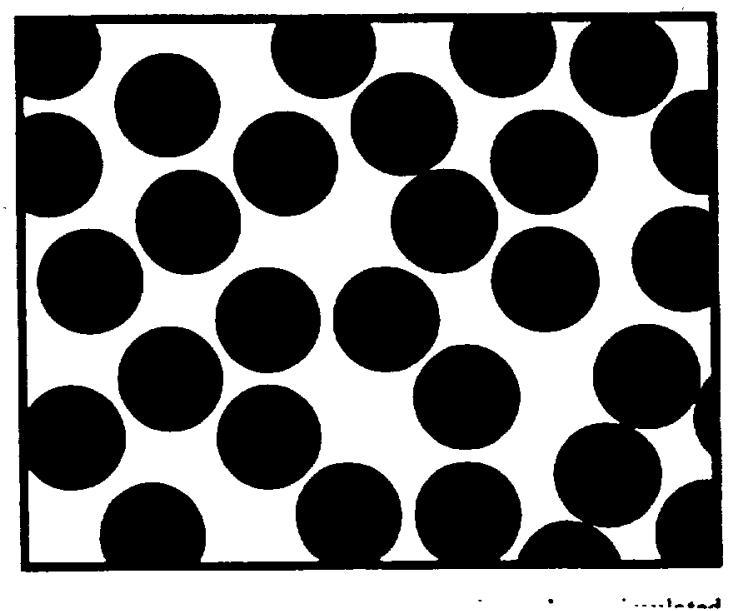




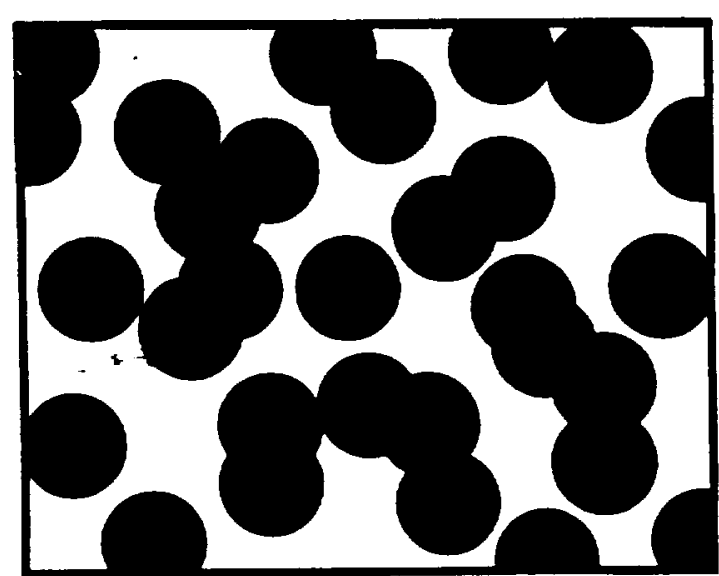

Fig. 2. A two-dimensional Boolean model microstructure.

second order attributes such as spatial arrangement of particles, rather than the first order parameters such as volume fraction. Thus, a quantitative comparison of the relevant aspects of spatial arrangement of particles in the true and simulated microstructures is essential for choosing a specific model to represent a given microstructure. The spatial arrangement of particle section centers in a random plane through three-dimensional simulated microstructure can be quantified in a straight-forward manner. A comparison with the corresponding attributes measured on a random metallographic plane through true microstructure provides an objective criterion to verify whether a simulation model closely represents the true microstructure. The three-dimensional spatial arrangement of particles in true microstructure can then be approximated by the corresponding arrangement in the simulated three-dimensional microstructure: this is the main objective of the computer simulations in the present study.

\section{Descriptors of spatial arrangement of particle centroids}

An important descriptor of the spatial distribution of particle centroids is the nearest neighbor distribution function [2]. It is described by the probability density function $P(R)$ such that $P(R) \cdot \mathrm{d} R$ is the probability that there is no other particle centroid in a sphere of radius $R$ around an arbitrary particle, and that there is at least one particle centroid in the spherical shell of radii $R$ and $(R+\mathrm{d} R)$. In a metallographic plane (two-dimensional section) through three-dimensional microstructure, the nearest neighbor distribution function of particle section centers is defined by the probability density function $\delta(r)$ such that $\delta(r) \cdot \mathrm{d} r$ is the probability that there is no other particle section center in a circle of radius $r$ around an arbitrary particle section, and that there is at least one particle section center in a circular shell of radii $r$ and $(r+d r)$.

Another important descriptor of the spatial distribution of particle centroids is the radial distribution function [2-4]. It is described by the function $G(R)$ such that $\pi R^{2} \cdot N_{v} \cdot G(R) \cdot \mathrm{d} R$ is equal to the average number of particle centroids in a spherical shell of radii $R$ and $(R+\mathrm{d} R)$ around an arbitrary particle centroid. $N_{v}$ is the average number of particle centroids per unit volume of three-dimensional microstructure. Therefore, for a completely random distribution of point particles, $G(R)$ is equal to one. Analogously, the radial distribution function in a metallographic plane, $g(r)$, is defined such that $2 \pi r \cdot N_{A} \cdot g(r) \cdot d r$ is equal to the average number of particle section centers in a circular shell of radii $r$ and $(r+\mathrm{d} r)$ around an arbitrary particle section. $N_{\mathrm{A}}$ is the average number of particle section centers per unit area of metallographic plane.

A practical and efficient digital image analysis technique is available for experimental estimation of the radial distribution function $g(r)$ and the nearest neighbor distribution $\delta(r)$ in a metallographic plane [5]. These functions can also be calculated for a random plane through simulated three-dimensional microstructure. If the simulated microstructure is a good approximation of the true microstructure, then experimental measurements of $g(r)$ and $\delta(r)$ functions must be in good agreement with those calculated for the simulated modeled microstructure. In such a case, the connectivity and other attributes of connectedness of the particles in true three-dimensional microstructure (which cannot be measured) can be approximated by the corresponding values for the simulated modeled three-dimensional microstructure (which can be calculated). This is the approach adopted in the present investigation.

\section{EXPERIMENTAL WORK}

\section{Material}

The experiments were performed on a series of specimens of polymer matrix composite consisting of hollow spherical carbon particles dispersed in a hardened polymer resin matrix of Araldite. The particulate volume fraction was varied from 0.16 to 0.47 , but the particle size frequency function was the same for all the specimens. The hollow spherical carbon particulate powder was supplied by Carbosphere Inc.; the average particle diameter was $10.6 \mu \mathrm{m}$. The liquid polymeric resin (Araldite) and the hardener were supplied by Ciba Geigy. The appropriate proportions of the particulate powder, the liquid Araldite resin and the hardener were thoroughly mixed to ensure uniform distribution of the carbon particles, and the mixture was heated in vacuum to degas and to decrease the viscosity of the polymer. The molds were slowly rotated during the hardening period of the polymer (which is approximately $10 \mathrm{~h}$ ) to avoid any segregation of the particles due to gravitational effects.

\section{Electrical conductivity measurements}

The resistivity (and therefore, the conductivity) was measured by using the well-known "three point" 


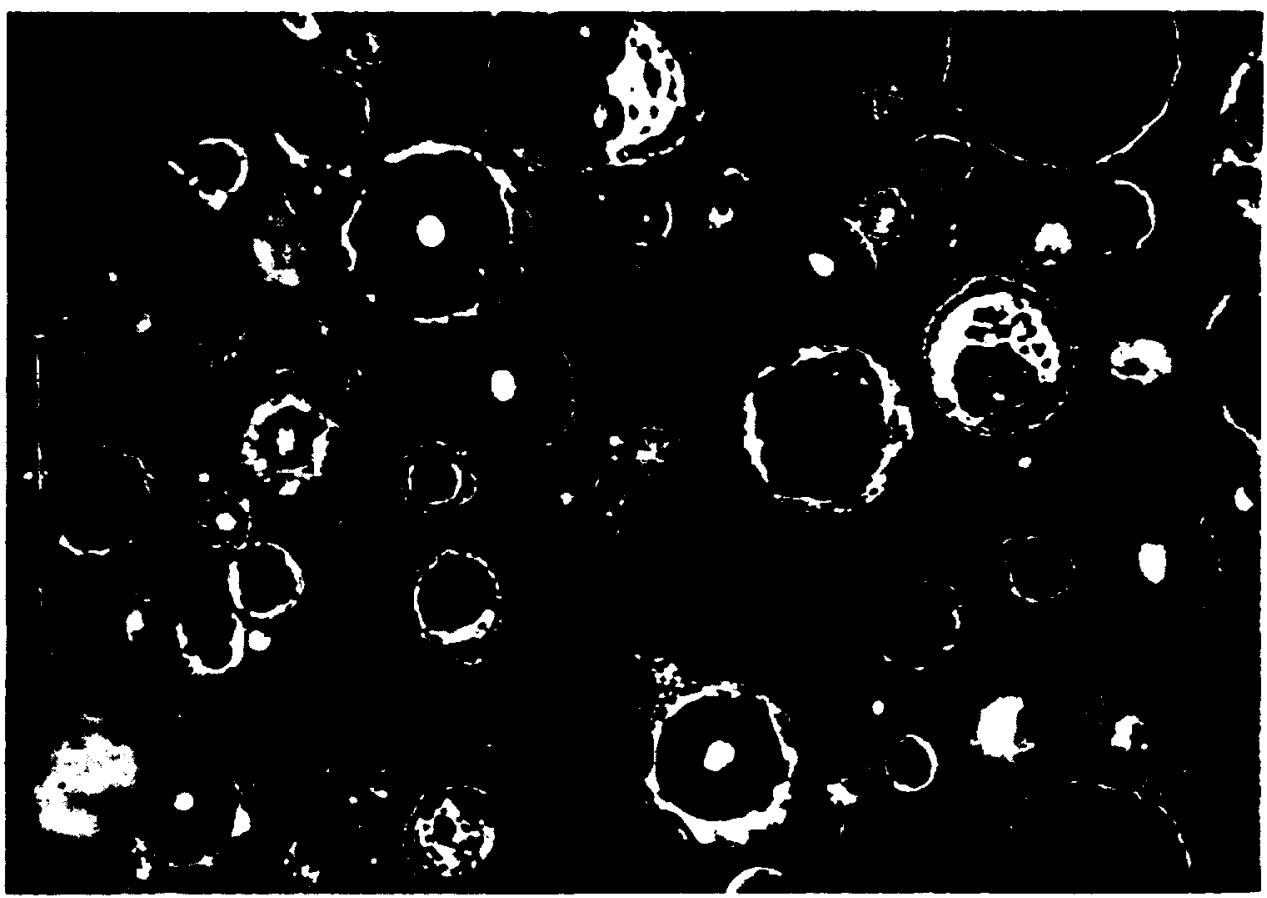

Fig. 3. Microstructure of a typical composite specimen observed on a representative metallograhic plane (particulate volume fraction $=0.29$ ); magnification, $500 \times$.

resistivity measurement technique. Cylindrical specimens of $14.25 \mathrm{~mm}$ diameter and $20 \mathrm{~mm}$ length were used for the conductivity measurements. Silver based conductive paint was coated on both the flat ends of the specimens, and a constant electrical current was applied across these two ends. A $1 \mathrm{~mm}$ wide circular ring was painted (with conductive paint) at about one fourth of the specimen length from each flat end, and the potential drop was measured across these two segments. The conductivity was calculated from the measured potential drop and the known constant applied current.

\section{Metallography}

The specimens were cut, mounted and polished on a series of $\mathrm{SiC}$ polishing papers, and then followed by polishing on diamond cloths on automatic polishing wheels. Figure 3 shows a typical microstructure revealed in this manner. Note that the carbon particles are round, and there is a range of particle sizes. In Fig. 3, the dark regions represent the "hollows" created by intersections of polishing plane with the hollow carbon particles; the bright regions are due to the near tangential intersections.

\section{Digital image analysis}

In this material, the contrast between the particles and the matrix is not very good, and therefore several image editing steps are necessary to develop the appropriate image contrast for subsequent measurements on the digitized images [12]. The image analysis was carried out on the images at the microscope magnification of $500 \times$. At this magnification, the observed particle sizes are about 50 pixels, which allows the measurements of particle sizes and interparticle distances with an error of less than $\pm 5 \%$. The measurements were performed on more than 100 fields of view on each specimen to ensure sampling of at least 3000 particles from each specimen. The centroid coordinates (referred to the same origin) and the particle section size of each particle were measured using an image analysis procedure described in detail elsewhere [5]. From these data, the radial distribution function and nearest neighbor distribution function of the particle section centroids in the metallographic plane were estimated (see Ref. [5] for details). These descriptors quantify the spatial arrangement of the particle sections in the metallographic plane. Figures 4 and 5 show the radial

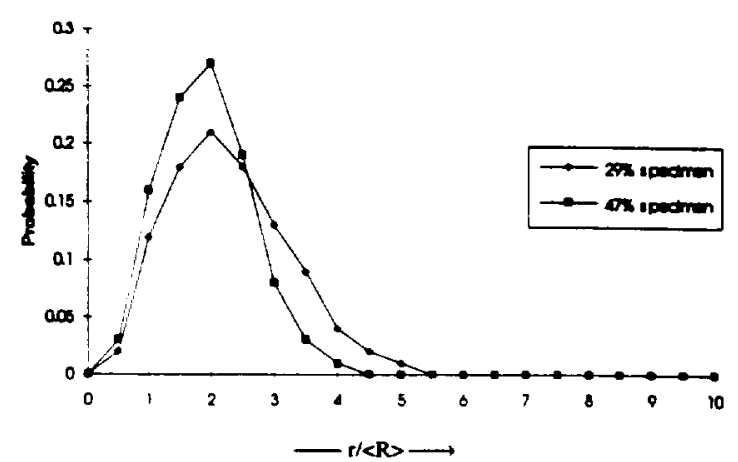

Fig. 4. Nearest neighbor distribution function in metallographic plane. 


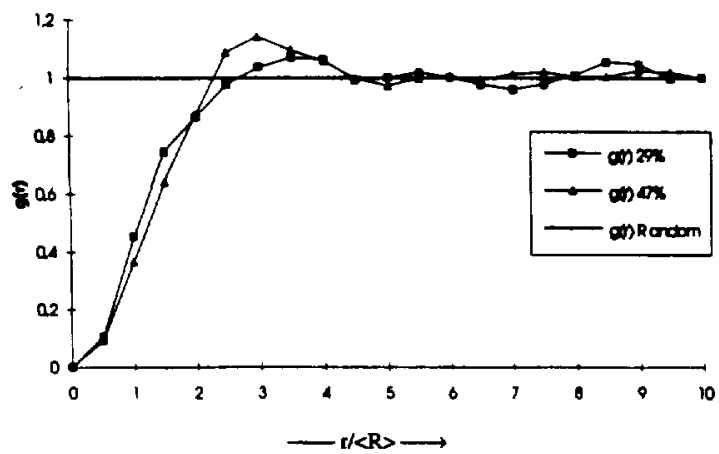

Fig. 5. Radial distribution function in metallographic plane.

distribution function and the nearest neighbor distribution function obtained in this manner. The particle section sizes in the metallographic plane were cast into the section size distribution, and the true threedimensional particle size distribution was estimated from these data by using stereological techniques [13]. Figure 6 shows the true particle size distribution estimated in this manner together with the size distribution of the carbon particulate powder measured directly by laser granulometry. An excellent agreement between the two distributions shows that the measurements on about 3000 particles yields an excellent representative sampling of the microstructure.

\section{NEW COMPUTER SIMULATED MICROSTRUCTURE MODEL}

In the microstructure of the present composite, the carbon particles are uniformly distributed in the matrix. These particles may (and do) touch one another, but they do not overlap significantly, i.e. they remain rigid spheres. A valid microstructure model must allow for these simple geometric aspects of the true microstructure. It follows that the "hard core sphere" model, where the particles are not allowed to touch one another (see Fig. 1) is not applicable to the present material. The Boolean mode] [9], which permits extensive overlaps among particles (see Fig. 2) is also not applicable. In the new model described below, the particles are first generated according to the Boolean model, which enables uniform distribution of particles. In the subsequent step, the overlapping particles are pulled apart from one another according to a repulsion law that allows maximum overlap up to one tenth of the particle diameter. This leads to a microstructure having uniformly distributed spherical particles that may have inter-particle contacts but no significant particle overlaps. Figure 7 shows a two-dimensional section through a typical three-dimensional microstructure simulated in this manner. It is worthwhile to compare Fig. 7 with the schematic microstructures shown in Figs 1 and 2. The details of the simulation steps of the new model are as follows.
(1) The volume of the three-dimensional microstructural space to be generated is specified depending on the average sphere size; typically, a cubic microstructural volume having edge length of approximately $10-30$ times average particle diameter is sufficient to obtain meaningful statistical averages. The simulated microstructural volume is periodic, and therefore, there is no edge effect.

(2) The size frequency function of the spheres is specified in terms of a histogram representation having 50 regularly spaced size classes. The absolute number of spheres in a given size class is directly proportional to the particle volume fraction to be simulated.

(3) The particles are then generated one by one according to the Boolean model [9], where the particle centroids are randomly located in the microstructural space and particles are allowed to freely overlap.

(4) The overlap vector of a pair of overlapping $i$ th and $j$ th particles $\mathbf{V}_{i j}$ is defined as the shortest vector displacement of the centroid of the $i$ th particle necessary to completely separate the overlapping $i$ th and $j$ th particles.

(5) The particles are analyzed for overlap one by one. Suppose that there are a total of $N$ particles and the $n$th particle is being analyzed, then its overlaps with only the next $(N-n)$ particles in the queue are analyzed; the overlaps with the first $(n-1)$ particles were analyzed when these particles were under attention. This scheme eliminates redundancy and makes the computation more efficient.

(6) Suppose the $n$th particle overlaps with the $i$ th, $j$ th and $k$ th particles (where, $i, j$ and $k>n$ ), then the net displacement vector of the $n$th particle, $V_{n}$ is given by the following equation.

$$
\mathbf{V}_{n}=\left[\mathbf{V}_{n i}+\mathbf{V}_{n j}+\mathbf{V}_{n k}\right] / M \text {. }
$$

The three displacement vectors on the right-hand side of equation (1) pertain to the overlap vectors for the particle pairs $n$ and $i, n$ and $j$, and $n$ and $k$, as defined in step (4). Repulsion strength, $M$ is one of the parameters of the model. In the present study, $M$ was set to be equal to 2 . Figure 8 illustrates the geometry involved in equation (1).

(7) The net displacement vector [step (6)] is calculated for all the overlapping particles in the system,

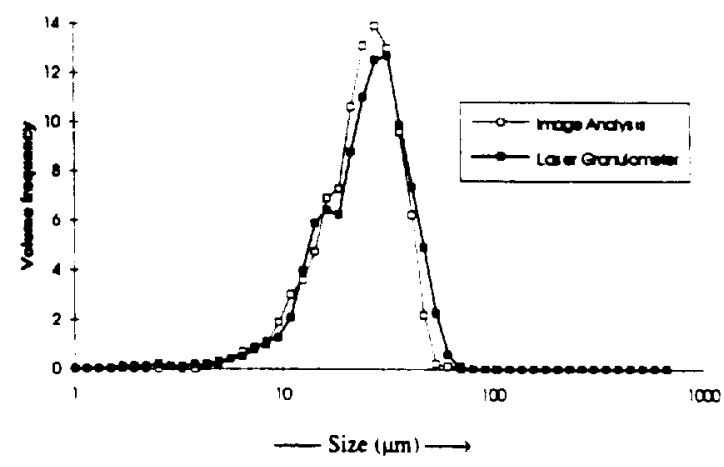

Fig. 6. Size distribution of carbon particles. 


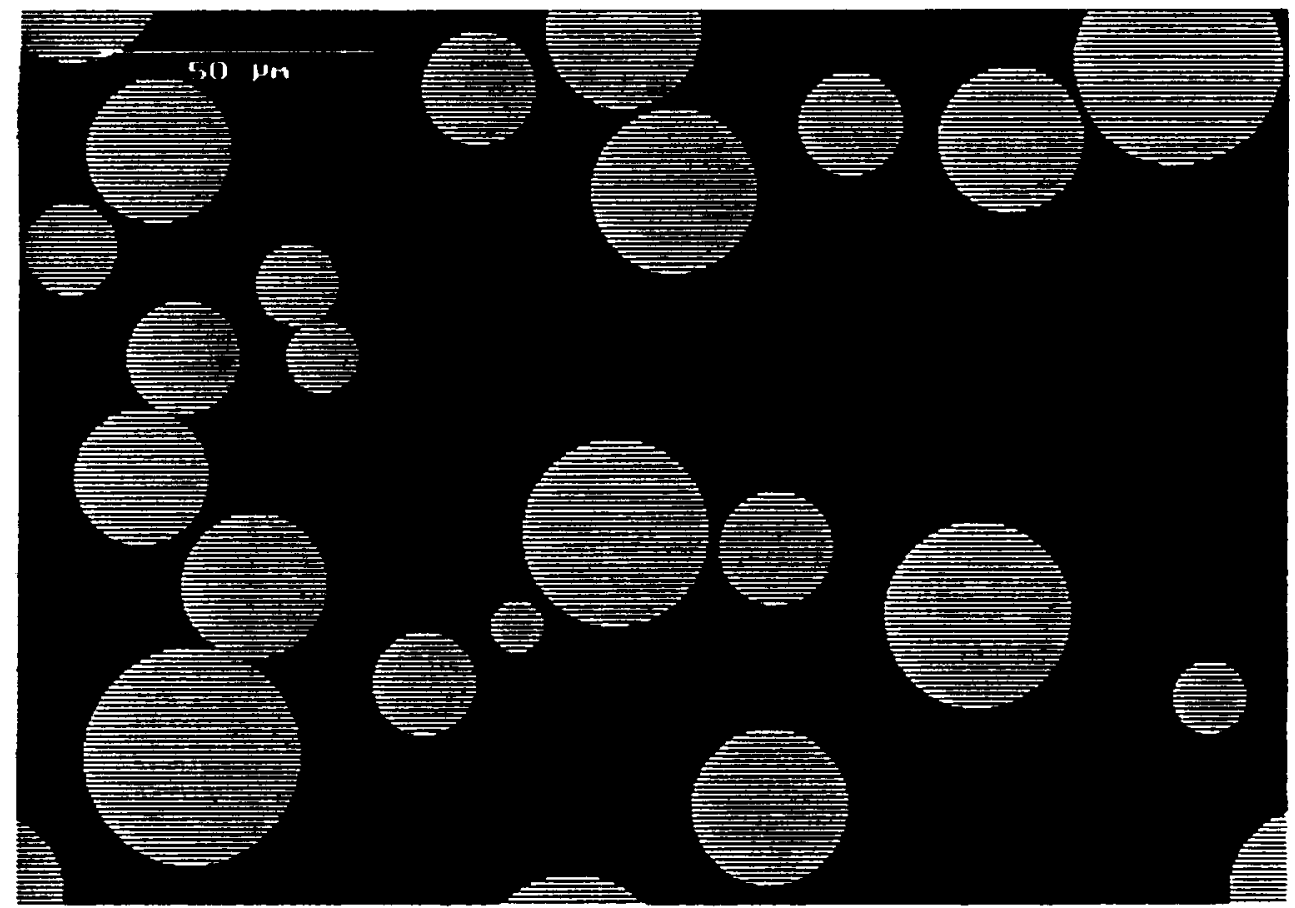

Fig. 7. Two-dimensional section through typical microstructure simulated from new model. Note that the particles touch, but do not overlap significantly.

and the centers of these particles are shifted by the corresponding net vector displacements.

(8) After the overlapping particles are displaced by their corresponding net vector displacements, the overlap vectors of all the overlapping particle pairs (in the new spatial arrangement) are recalculated, and

a)

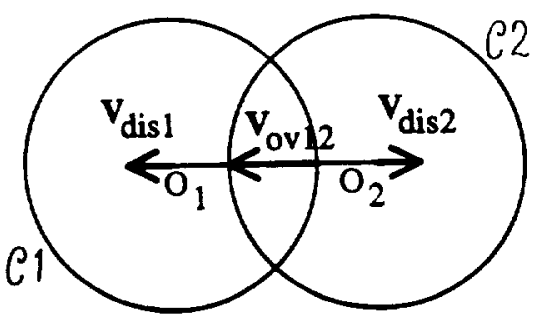

b)

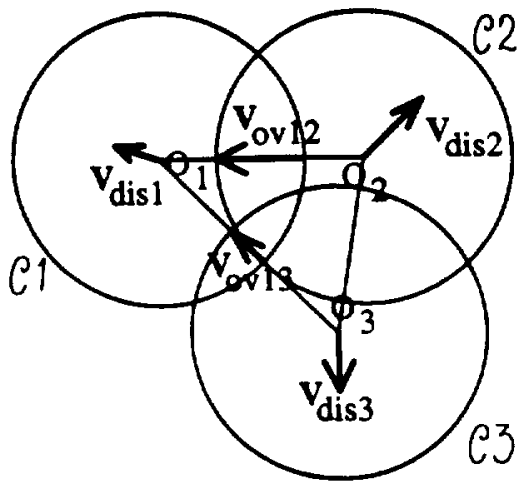

Fig. 8. Definition of the net displacement vector. the magnitude of maximum particle overlap, $\tau$, is calculated.

(9) The maximum permissible particle overlap, $\epsilon$, in the final microstructure is an input parameter of the model whose value has to be specified. In the present case, $\epsilon$, was set to be equal to one tenth of the average particle diameter. If $\tau>\epsilon$ then, the steps $(5)-(8)$ are repeated as the next iteration of the simulation. These iterations are continued until the actual maximum overlap $\tau$ reaches a value lower than the set maximum value $\epsilon$; at that point the simulation is terminated. Figure 7 shows a two-dimensional section through a typical three-dimensional microstructure simulated in this manner.

\section{Adjustable model parameters}

The maximum permissible particle overlap, $\epsilon$, and the repulsion strength, $M$, are the adjustable parameters of the model. The rate of convergence of the simulation (i.e. number of iterations after which actual maximum overlap, $\tau$ is less than $\epsilon$ ) depends on these two parameters. As $t$ approaches zero, the necessary number of iterations approaches infinity. In such a limiting case, the simulated microstructure reaches a stage where only tangential point contacts between the particles are allowed. A very large value of $t$ leads to a simulated microstructure having extensive particle overlaps (as in the Boolean model), which is not realistic in the present context. A value of $t$ equal to one-tenth of the average particle diameter yields microstructure that is close to the actual microstructure, and permits completion of simulation 


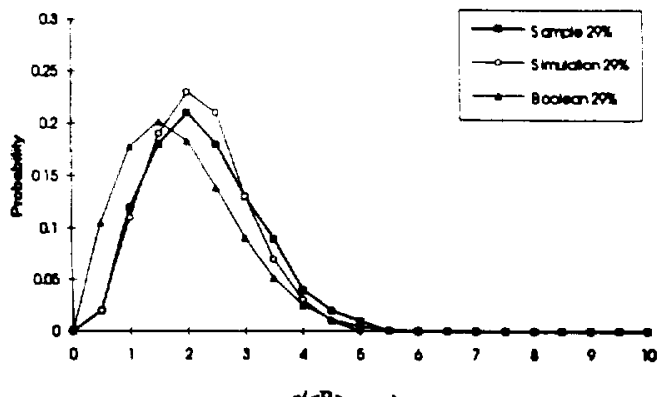

b)

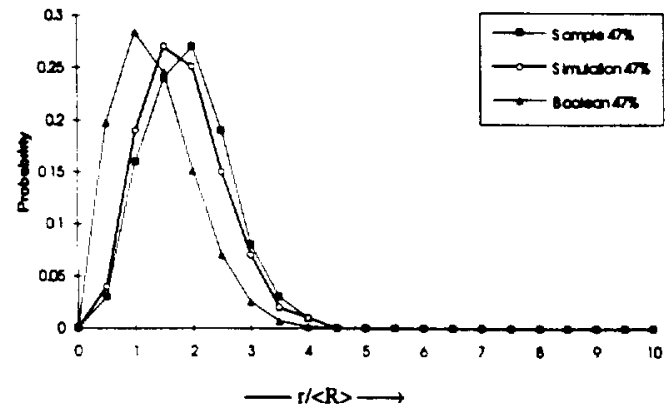

Fig. 11. Experimental and simulated nearest neighbor distribution function on a two-dimensional section.

one face of the specimen to another face. If there is not a single particle chain connecting the two faces of the specimen, then the conductivity is expected to be zero. Thus, there is a microstructural percolation threshold: there exists a critical volume fraction of the particles $f^{*}$ below which the probability of the formation of a continuous particle chain is almost zero. and consequently the electrical conductivity is also zero. This critical volume fraction $f^{*}$ depends on the size distribution of the particles.

As the particle volume fraction $f$ increases beyond $f^{*}$, there are more continuous pathways for the electrical conduction, and more particles participate in the conduction process. In this stage, the conductivity is a direct function of the volume fraction of the particles that actually participate in the conduction process. This is illustrated in Fig. 12. In this figure, the bright particles are not connected to any chain, and therefore they do not participate in the conduction process. The black particles that form continuous chains contribute to the electrical conduction. The cross-hatched particles touch the chains formed by black particles, but they do not contribute to the electrical conductivity. A particle actively participates in the electrical conduction process if and only if from that particle one can travel through the particulate phase in at least two distinctly different directions (each path through different inter-particle contact belonging to the same particle) and reach the two external surfaces of the specimen across which an electrical potential is applied, without ever leaving the particulate phase. Therefore, the most simple microstructural parameter that is relevant to the electrical

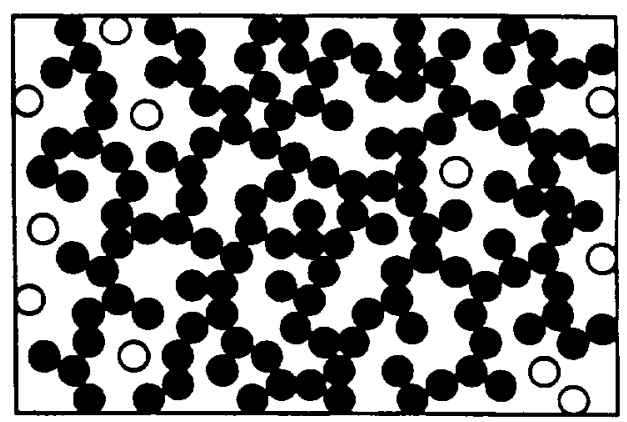

Particles that belong to the main cluster and effectively participate to the electrical conductivity

Particles that belong to the main cluster but do not participate to the electrical conductivity

Particles that do not belong to the main cluster

Fig. 12. Identification of the particles that take part in the electrical conduction

conduction process is the volume fraction $f_{c}$ of only those particles that actively participate in the conduction process according to this definition. To the best of our knowledge, there is no experimental technique to identify in the material microstructure the particles that are effective in the conduction process, and therefore the effective volume fraction $f_{c}$ cannot be experimentally measured. However, such particles can be identified in a computer simulated microstructure, and their volume fraction $f_{\mathrm{c}}$ can be calculated. It has been shown that the overall spatial arrangement of the particles in our simulated microstructures is a reasonable approximation for that in the corresponding actual microstructures (see Figs 9-11). Thus, the effective volume fraction $f_{c}$ in the real microstructure can be approximated by its value in the simulated microstructure. Figure 13 shows a plot of the effective volume fraction $f$ versus the overall particulate volume fraction $f$. The percolation volume fraction $f^{*}$ is equal to 0.26 . Note that for $f<f^{*}$, $f_{\mathrm{r}}=0$. There is a discontinuous jump in the effective volume fraction. $f_{\mathrm{c}}$ at $f=f^{*}$. As the overall particulate volume fraction $f$ increases beyond the percolation volume fraction $f^{*}$. the effective volume fraction

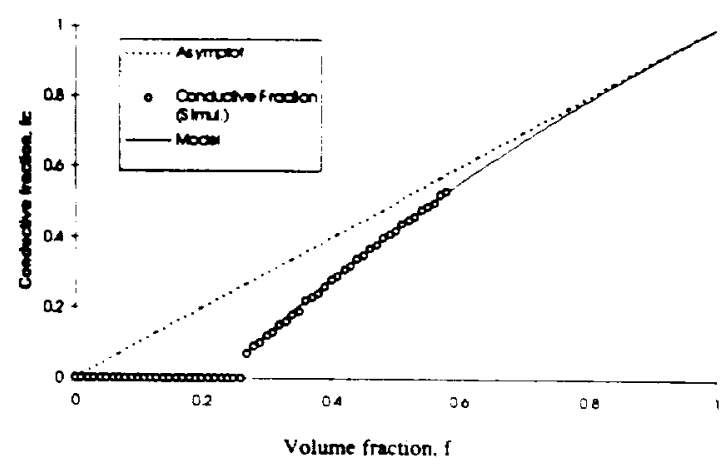

Fig. 13. Plot of effective volume fraction $f$ versus the overall particulate volume fraction. $t$. 
$f_{c}$ approaches the overall particulate volume fraction $f$. The following empirical equation represents the computed data in Fig. 13 quite well

$$
f_{c}=2.78(f+0.21)^{0.4}-2.0 \text { for } f>f^{*} \text {. }
$$

The relationship between the effective volume fraction and overall global volume fraction $f$, is the necessary input for the self-consistent model for electrical conductivity discussed below.

\section{MODEL FOR ELECTRIC CONDUCTIVITY}

There are a number of theoretical treatments that predict the bounds on the electrical conductivity of two-phase composites. The most simple treatment is the one due to Voigt and Reuss [14]. The composite has maximum possible conductivity when the two phases are stacked in the form of alternate parallel slabs extending from one end of the specimen to another, and the electrical current is applied parallel to the slabs; the conductivity is minimum when the applied current is perpendicular to the slabs (the two arrangements can be visualized as "series" and "parallel" configurations of the resistors). These bounds depend only on the volume fraction of the two phases. Hashin and Strikman [15] have given tighter bounds than those of Voigt and Reuss, however, these bounds are also completely determined by volume fraction of the two phases.

Landauer [16] has given a model for the estimation of electrical conductivity of two-phase composites where the two phases are randomly distributed and isotropic (i.e. no preferred orientation). Although, Landauer's derivation is based on the assumption that the particulate has a spherical shape, the resulting expression for electrical conductivity is expected to be a good approximation for any two-phase isotropic microstructure having random distribution of the two phases. Landauer [16] has given the following expression for estimation of the electrical conductivity, $P$ of a two-phase composite material containing particulate

$$
\begin{gathered}
P=\left[\left(3 f_{1}-1\right) \cdot P_{1}+\left(3 f_{2}-1\right) \cdot P_{2}+\left\{\left[\left(3 f_{1}-1\right) \cdot P_{1}\right.\right.\right. \\
\left.\left.\left.+\left(3 f_{2}-2\right) \cdot P_{2}\right]^{2}+8 \cdot P_{1} \cdot P_{2}\right\}^{1 / 2}\right] / 4 .
\end{gathered}
$$

In equation (3), $P_{1}$ and $P_{2}$ are the electrical conductivities of the particulate and the matrix, respectively, and $f_{1}$ and $f_{2}$ are their volume fractions. Obviously,

$$
f_{1}+f_{2}=1
$$

In the Landauer's model it is assumed that all the particles contribute to the electrical conduction, which is reasonable if the conductivities of the particulate and the matrix are not drastically different. However, in the present composite, the conductivities of the carbon particles and the resin matrix differ by sixteen orders of magnitude. Therefore, a particle can contribute to the electrical conduction if and only if there is a continuous path that goes through two different inter-particle contacts of the same particle, and connects opposite faces of the specimen across which an electrical potential is applied (see Fig. 12). The particles that do not satisfy this condition do not contribute to the conduction process, and hence they can be regarded as a part of the matrix, for estimation of the electrical conductivity. Therefore, for the present composite, the volume fractions $f_{1}$ and $f_{2}$ in equation (3) must be appropriately interpreted: $f_{1}$ should be equal to the effective particulate volume fraction $f_{c}$, and $f_{2}$ is equal to $\left(1-f_{c}\right)$. Further, in the present case, the electrical conductivity of the matrix, $P_{2}$ can be equated to zero. With these changes, equation (3) takes the following modified form

$$
\begin{gathered}
P=0 \text { for } 0 \leqslant f_{c} \leqslant 1 / 3 \\
P=\left(3 f_{c}-1\right) \cdot P_{1} / 2 \text { for } 1 / 3 \leqslant f_{c} \leqslant 1 .
\end{gathered}
$$

Equation (5) predicts that the electrical conductivity should increase linearly with the effective particulate volume fraction, for $f_{c}>1 / 3$, and it should be equal to zero for the effective volume fraction less than $1 / 3$. Substituting the value $f_{c}$ equal to $1 / 3$ in equation (2) yields, $f=0.42$ for the percolation threshold for electrical conduction. Recall that morphological percolation threshold for the initiation of the formation of continuous particle chains (i.e. $f^{*}$ ) occurs at the particle volume fraction equal to 0.26 . Therefore, the morphological percolation threshold and the percolation threshold for electrical conduction predicted by Landauer's equation are not equal. The morphological percolation threshold gives the particulate volume fraction at which the continuous particle chains begin to form. At this stage, the conductivity is non-zero in one direction (where a continuous chain has formed), and it is zero in all other directions. However, for isotropic electrical conduction, it is essential to have a number of continuous particle chains randomly oriented in three-dimensional space, and this is achieved only at a volume fraction higher than that for morphological percolation threshold. Landauer's equation assumes isotropy in electrical conduction. and therefore, it is applicable only after a sufficient number of continuous particle chains are formed. Due to this reason, Landauer's equation predicts a percolation threshold for electrical conduction $(f=0.42)$ that is higher than the morphological percolation threshold for the formation of continuous particle chains $(f=0.26)$.

Equation (5) predicts that for $f_{c}>1 / 3$, the electrical conductivity varies linearly with the effective particulate volume fraction $f_{c}$. Further the slope of the linear portion of the plot should give the effective conductivity of the particulate phase. Figure 14 shows the plot of experimentally measured electrical conductivity vs the effective particulate volume fraction $f_{c}$. The values of $f_{c}$ were obtained from the global volume fraction $f$ by using the curve in Fig. 13 . obtained from computer simulations. The data is in good agreement with the predictions of equation (5). 


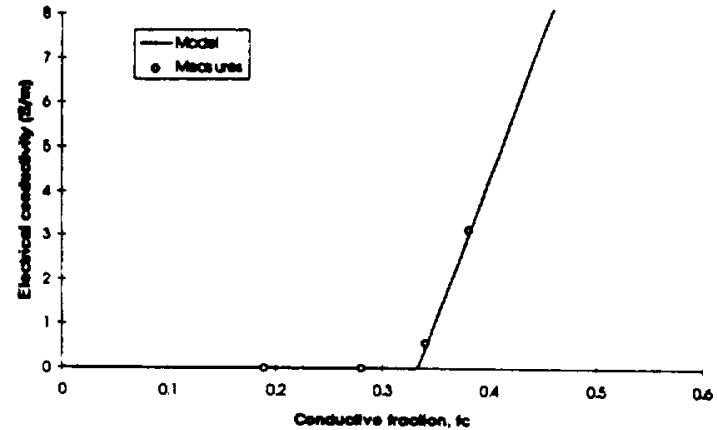

Fig. 14. Plot of electrical conductivity versus effective volume fraction $f_{c}$.

The conductivity is zero for effective particulate volume fraction less than about 0.33 , as predicted. However, the slope of the linear region of the plot yields $43 \mathrm{~S} / \mathrm{m}$ for the effective conductivity of the carbon particulate phase, which is lower than the reported bulk conductivity of carbon $(200 \mathrm{~S} / \mathrm{m})$ by a factor of five or so. This is not surprising, because the inter-particle contact resistance is expected to be significantly higher than the bulk electrical resistance. Thus, the effective conductivity of the particulate reflects the conductivity of the inter-particle contacts rather than the bulk conductivity of carbon. It follows that the calculated value of $43 \mathrm{~S} / \mathrm{m}$ essentially represents the conductivity of the inter-particle contacts, and therefore it is reasonable.

\section{CONCLUSIONS}

Computer simulation of a three-dimensional microstructure is useful to quantify the microstructural attributes associated with the "connectedness" that are difficult to measure experimentally. In this study, computer simulated three-dimensional microstructures were used to estimate the morphological percolation volume fraction for electrical conduction, and particulate volume fraction that is effective in the electrical conduction in a polymer matrix composite. These calculated values are successfully utilized to predict the conductive particulate volume fraction dependence of the electrical conductivity in a polymer matrix composite where the matrix is dielectric.

Acknowledgements - This research was conducted at Georgia Institute of Technology during LP's tenure as a visiting scientist, sponsored by Aerospatiale, France. LP gratefully acknowledges the financial support from Aerospatiale. AMG gratefully acknowledges the financial support from Microgravity Science Applications Division, N.A.S.A. (grant number NAG3-165I), and U.S. National Science Foundation (grant number DMR-9301986) for his contribution to this research.

\section{REFERENCES}

1. J. P. Jernot, B. Prasad and P. Demaleprade, J. Microsc. 167,9 (1992).

2. B. D. Ripley, Spatial Statistics. John Wiley and Sons, London (1981).

3. K. H. Hanish, Biometrics J. 25, 731 (1983).

4. C. W. J. Beenakker, Phys. Rer. 33, 4482 (1986)

5. P. Louis and A. M. Gokhale, Metall. Mater. Trans 26A, 1449 (1995).

6. K. H. Hanish and D. Stoyan, J. Microsc. 122, 131 (1980).

7. K. H. Hanish, D. Konig and D. Stoyan, J. Microsc. 140, 361 (1985).

8. D. Jeulin, Proc. Presidential Symposium on Microbeam Analysis, Milwaukee, Wisconsin (1988).

9. J. Serra, J. Microse. 156, 124 (1989).

10. P. Meakin and R. Jeulin, Europhys. Lett. 9, 71 (1989).

11. D. W. Cooper, Phys. Rer. 38, 522 (1988).

12. J. Serra, Image Analysis and Mathematical Morphology Academic Press, London (1988).

13. R. T. DeHoff and F. N. Rhines, Quantirative Microscopy. McGraw-Hill, New York (1968).

14. P. L. Rossiter, The Electrical Resistivity of Metals and Alloys. Cambridge University Press, Cambridge, U. K. (1987).

15. Z. Hashin and S. Shtrikman, J. appl. Phys. 33, 3125 (1962).

16. R. Landauer, J. appl. Phys. 23, 779 (1952). 